\title{
Does metabolic control affect salivary adipokines in type 2 diabetes mellitus?
}

\section{Czy kontrola metaboliczna cukrzycy typu 2 ma wpływ na adipokiny w ślinie?}

\author{
Elif Teke ${ }^{1, A, B, D}$, Fatma Yeşim Kırzıoğlu ${ }^{1, A, C-F}$, Hakan Korkmaz ${ }^{2, A, B, D-F}$, Mustafa Calapoğlu ${ }^{3, A-C}$, Hikmet Orhan ${ }^{4, C}$ \\ ${ }^{1}$ Department of Periodontology, Faculty of Dentistry, Süleyman Demirel University, Isparta, Turkey \\ ${ }^{2}$ Department of Internal Medicine, Faculty of Medicine, Süleyman Demirel University, Isparta, Turkey \\ ${ }^{3}$ Department of Biochemistry, Faculty of Arts and Sciences, Süleyman Demirel University, Isparta, Turkey \\ ${ }^{4}$ Department of Biostatistics and Medical Informatics, Faculty of Medicine, Süleyman Demirel University, Isparta, Turkey \\ A - research concept and design; $\mathrm{B}$ - collection and/or assembly of data; $\mathrm{C}$ - data analysis and interpretation; \\ $D$ - writing the article; $E$ - critical revision of the article; $F$ - final approval of the article
}

\section{Address for correspondence \\ Fatma Yeşim Kırzıoğlu}

E-mail: fyesimbozkurt@gmail.com

\section{Funding sources}

None declared

Conflict of interest

None declared

\section{Acknowledgements}

This study was supported by the Scientific Research Foundation of Süleyman Demirel University, Isparta, Turkey (project No. 4930-DU1-17).

Received on December 27, 2018 Reviewed on December 30, 2018 Accepted on January 28, 2019

Published online on March 29, 2019

Cite as

Teke E, Kırzıoğlu FY, Korkmaz H, Calapoğlu M, Orhan H. Does metabolic control affect salivary adipokines in type 2 diabetes mellitus. Dent Med Probl. 2019;56(1):11-20. doi:10.17219/dmp/103417

DOI

10.17219/dmp/103417

Copyright

○ 2019 by Wroclaw Medical University

This is an article distributed under the terms of the

Creative Commons Attribution 3.0 Unported (CC BY 3.0)

(https://creativecommons.org/licenses/by/3.0/)

\begin{abstract}
Background. Adipokines produced by adipose tissue initiate pro-inflammatory events and contribute to the pathogenesis of diabetic periodontitis.

Objectives. The aim of this study was to evaluate the effect of the metabolic status on the level of salivary adipokines in type 2 diabetes mellitus (T2DM) patients.
\end{abstract}

Material and methods. A total of 239 individuals, including 161 T2DM patients and 78 healthy $(\mathrm{H})$ controls, participated in the study. The metabolic control status was evaluated in each person. Periodontal measurements were recorded. Periodontal epithelial surface area (PESA), periodontal inflamed surface area (PISA) and the total dental index (TDI) were calculated. The salivary adiponectin, tumor necrosis factor a (TNF-a), interleukin 6 (IL-6), and vaspin levels were determined.

Results. The T2DM patients had higher periodontal parameters and adiponectin, TNF-a, IL-6, and vaspin levels as compared with the $\mathrm{H}$ controls $(p<0.05)$. As the metabolic control worsened, periodontal pocket depth (PPD) and clinical attachment level (CAL) increased. When covariates (age, gender, body mass index - BMI, education level, smoking, dental visit and tooth brushing frequency) were adjusted, only the TNF-a and vaspin levels were significantly higher in the T2DM patients $(p<0.05)$. In the T2DM patients, positive correlations were found between the TNF-a level and the percentage of bleeding on probing (BOP\%), PPD, PESA, and PISA, and between the adiponectin level and PISA. Moreover, there was a negative relationship between the salivary volume and TDI. While the correlations IL-6-TNF- $a$, vaspin-triglycerides and vaspin-tooth brushing frequency were positive, the statistically significant associations vaspin-IL-6 and vaspin-low-density lipoprotein $(L D L)$ were negative $(p<0.05)$.

Conclusions. The severity of periodontal disease increases as the metabolic control status worsens. The levels of salivary adipokines were changed by T2DM, while being independent from the metabolic control.

Key words: adipokines, saliva, periodontal disease, diabetes mellitus type 2, vaspin

Słowa kluczowe: adipokiny, ślina, choroba przyzębia, cukrzyca typu 2, waspina 


\section{Introduction}

Type 2 diabetes mellitus (T2DM) is a disease characterized by a chronic hyperglycemic condition, which is caused by the apparent or relative lack of insulin release and/or the effect of released insulin. As a result of persistent hyperglycemia in T2DM, the proteins are irreversibly glycosylated, leading to advanced glycation end-products (AGEs). It has been suggested that an increased level of AGEs aggravates inflammatory tissue destruction by increasing cytokine secretion in response to periodontopathogens, and impair biological functions via the cell-cell and cell-matrix associations in connective tissue. ${ }^{1}$ An increase in the glycated hemoglobin $\mathrm{A}\left(\mathrm{HbA}_{1 \mathrm{c}}\right)$ levels, an indicator of glycemic control in T2DM, is related to the severity of periodontitis, and the control of periodontal disease is considered necessary for the management of T2DM. ${ }^{1-3}$

Adipocytes, also considered specific endocrine cells, produce many cytokines/adipokines that act as pro- (e.g., interleukin 6 - IL-6, tumor necrosis factor $\alpha-$ TNF- $\alpha$ ) or anti-inflammatory (e.g., adiponectin) agents and participate in inflammation. Adipokines are important in regulating some processes, including insulin resistance, energy balance, inflammation, blood pressure, hemostasis, and endothelial function, which play key roles in the mechanism of T2DM. ${ }^{4}$ The systemic TNF- $\alpha$ and IL-6 levels increase as a function of the severity of the 2 diseases in patients with periodontitis, with and without T2DM. Unlike other adipokines, the circulating concentration of adiponectin is low in obesity, hypertension, T2DM, and cardiovascular diseases. ${ }^{6}$ Vaspin is defined as an adipokine and may have a causative or protective effect in various inflammatory diseases. ${ }^{7}$

Saliva is a biological fluid that can be collected noninvasively. The mediators or biomarkers in saliva indicate oral and general health. ${ }^{8}$ This study hypothesized that there exists a relationship between the metabolic control and the levels of salivary adipokines in T2DM patients. Therefore, the effect of the metabolic status on the salivary adipokine levels in T2DM patients was evaluated and their clinical periodontal parameters were compared with those of systemically healthy $(\mathrm{H})$ individuals.

\section{Material and methods}

\section{Subject selection}

This study was conducted in accordance with the ethical standards of the Helsinki Declaration (1975, revised in 2002). It was approved by the Clinical Research Ethics Committee, Süleyman Demirel University, Faculty of Medicine, Isparta, Turkey (March 1, 2017/4).
Of the 360 individuals who came to the diabetes clinic between March 2017 and February 2018, a total of 239 (161 T2DM patients and $78 \mathrm{H}$ individuals) participated in this study. All of them signed an informed consent form.

The criteria adopted for inclusion in the study were the following: age >35 years; at least 8 natural teeth, excluding third molars; no periodontal intervention $\leq 6$ months prior to the study; and no antibiotics and/or anti-inflammatory drugs $\leq 3$ months before the study. The systemic status of each participant was determined by detailed examinations and biochemical analyses. The T2DM patients were diagnosed with the disease according to the diagnostic criteria recommended by the American Diabetes Association (ADA). ${ }^{9}$ The participants were excluded if they presented with aggressive periodontitis, oral pathology or immunodeficiency, needed a premedication for dental treatment, were undergoing hormone replacement therapy or chemotherapy/radiotherapy, were pregnant/lactating, had an active infection, a rheumatic or malignant disease, were using drugs that cause gingival overgrowth (cyclosporin, etc.), and if diabetes diagnosis age was $<1$ year. The T2DM patients were divided into subgroups, based on the $\mathrm{HbA}_{1 \mathrm{c}}$ level regarding the metabolic control status (<7\%: good; $\geq 7 \%$ : poor). The $\mathrm{H}$ individuals did not have any systemic disease.

Data related to systemic conditions (blood pressure, body mass index - BMI, medications, and serum parameters: fasting blood sugar - FBS, $\mathrm{HbA}_{1 \mathrm{c}}$, total cholesterol - TC, low-density lipoprotein - LDL, high-density lipoprotein - HDL, triglycerides - TG, C-reactive protein - CRP, neutrophil-to-lymphocyte ratio - NLR, creatinine, alanine aminotransferase - ALT) were recorded. The participants were asked to complete a questionnaire that included questions about their sociodemographic characteristics and habits (oral care, smoking, etc.).

\section{Periodontal examination}

Periodontal measurements, as well as the plaque in$\operatorname{dex}(\mathrm{PI}),{ }^{10}$ the gingival index (GI) ${ }^{11}$ periodontal pocket depth (PPD), percentage of bleeding on probing (BOP\%), and clinical attachment level (CAL) were performed by 1 calibrated dentist (E.T.) using a William's periodontal probe (Hu-Friedy, Chicago, USA). Periodontal pocket depth and CAL were measured at 6 sites, whereas PI, GI and BOP\% were evaluated at 4 sites per tooth. Periodontal epithelial surface area (PESA) and periodontal inflamed surface area (PISA) of each individual were calculated in a Microsoft Excel spreadsheet, accessible online at www.parsprototo.info. ${ }^{2}$ The number of caries and periapical lesions were determined according to oral and radiographic evaluations, and the total dental index (TDI $)^{12}$ was calculated. The number of missing teeth was also recorded. 


\section{Salivary sampling}

Unstimulated total saliva samples were collected in the morning before the periodontal examination. Individuals were asked to lean forward in front of the test tube for $10 \mathrm{~min}$, allowing the passive release of saliva and the anticipation of an open mouth. ${ }^{13}$ The collected samples were stored at $-80^{\circ} \mathrm{C}$ until assayed. Before analysis, the saliva samples were stored at $-20^{\circ} \mathrm{C}$ for $12 \mathrm{~h}$, then warmed to $4^{\circ} \mathrm{C}$ for dissolution. After vortexing, they were centrifuged at $9000 \times \mathrm{g}$ for $6 \mathrm{~min}$ at $4^{\circ} \mathrm{C}$ and the resultant supernatants were collected.

\section{Adipokine measurements}

The salivary adiponectin, IL- 6 , vaspin, and TNF- $\alpha$ levels were determined by the sandwich enzyme-linked immunosorbent assay (ELISA) method, according to the manufacturer's recommendations (BMS2032/2 - Bender MedSystems GmbH, Vienna, Austria; BMS213/2 - Bender MedSystems GmbH; 201-12-0922 - Sunred Biological Technology Co., Inc., Shanghai, China; KAP1751 - DIAsource ImmunoAssays S.A., Ottignies-Louvain-la-Neuve, Belgium, respectively). The absorbance of the calibrators, controls and samples of all the tests performed was read at $450 \mathrm{~nm}$ and $630 \mathrm{~nm}$ (reference filter $550 \mathrm{~nm}$ or $650 \mathrm{~nm}$ ) in a microplate reader (Epoch ${ }^{\mathrm{TM}}$; BioTek Instruments, Inc., Winooski, USA). The optical densities of the samples in each well were converted to concentration by software-mediated comparison, via a standard curve through the same analytical run, using a 4-parameter curve-fitting software (http://www.myassays.com/).

\section{Statistical analyses}

The IBM SPSS v. 20.0 software (IBM, Chicago, USA) was used to evaluate the study findings. The Kolmogorov-Smirnov test was used for normal distribution suitability, in controlling the parametric assumptions of the variables. The homogeneity of the variances was assessed by Levene's test. The significance of the relationships between the categorical variables was examined by the $X^{2}$ independence test. The comparisons of the continuous variables, according to the groups, were analyzed by multivariate analysis of variance (MANOVA), with covariates (age, gender, BMI, education level, smoking, dental visit and tooth brushing frequency) unadjusted and adjusted. When statistical significance in the comparisons was found, the least significant difference multiple comparison test was used for binary comparisons of group means. The significance of the linear relationships between the periodontal, metabolic and salivary parameters was analyzed by Pearson's correlation $(r)$. The significance level was set at $p=0.05$.

\section{Results}

Based on the sociodemographic and anthropometric characteristics of the individuals (Table 1), there was a significant difference between the T2DM patients and $\mathrm{H}$ individuals $(p<0.05)$, except for gender. When individuals were grouped based on the metabolic control status, there were significant differences in all characteristics $(p<0.05)$, except for smoking. Diabetes diagnosis age and drug use were similar between the T2DM patients with good and poor metabolic control $(p>0.05)$.

Table 2 shows the metabolic parameters. It was found that the levels of FBS, $\mathrm{HbA}_{1 \mathrm{c}}$ and CRP, and the TC-to-HDL ratio (TC/HDL) were significantly higher in the T2DM patients than in the $\mathrm{H}$ individuals, and also higher in the T2DM patients with poor metabolic control than in the T2DM patients with good metabolic control $(p=0.000)$. When covariates (age, gender, BMI, education level, smoking, dental visit and tooth brushing frequency) were adjusted, the TC, TG and ALT levels were similar between the T2DM patients with good and poor metabolic control, and also the $\mathrm{H}$ controls $(p>0.05)$. Significantly higher systolic-diastolic blood pressures and NLR were demonstrated in the T2DM patients as compared with the $\mathrm{H}$ individuals $(p<0.001)$. The HDL levels were found to be lower in the T2DM patients than in the $\mathrm{H}$ controls, regardless of the metabolic control status $(p=0.000)$. Also, the mean CRP levels in the T2DM patients with poor metabolic control were different from those in both the T2DM patients with good metabolic control and the $\mathrm{H}$ individuals $(p<0.05)$. There was no significant difference between the T2DM groups regarding LDL and creatinine $(p>0.05)$.

Periodontal parameters (PI, GI, BOP\%, PPD, CAL), the number of missing teeth, PESA, PISA, and TDI scores were increased in the T2DM patients $(p<0.001)$. When covariates (age, gender, BMI, education level, smoking, dental visit and tooth brushing frequency) were adjusted, the differences in the oral parameters between comparisons continued, except for PI $(p<0.05)$. The salivary volume was decreased in the T2DM patients compared with controls, but independent from the metabolic control status $(p<0.05)$. Both PPD and CAL were higher in the T2DM group with poor metabolic control than in the T2DM group with good metabolic control, with covariates unadjusted, although the difference between the T2DM patients with good and poor metabolic control became statistically insignificant when covariates were adjusted $(p=0.05)$ (Table 3$)$.

The salivary adipokine levels are shown in Table 3. While the TNF- $\alpha$, IL- 6 and vaspin levels in saliva were increased in the T2DM subjects, regardless of the metabolic control status, adiponectin was decreased. Although the salivary adiponectin levels were higher in the $\mathrm{H}$ individuals than in the T2DM patients with poor 
Table 1. Comparisons of sociodemographic and anthropometric characteristics

\begin{tabular}{|c|c|c|c|c|c|c|}
\hline Variables & $\begin{array}{l}\text { Group } \mathrm{H} \\
\mathrm{n}=78\end{array}$ & $\begin{array}{l}\text { Group T2DM } \\
n=161\end{array}$ & $\begin{array}{l}\text { Good metabolic control } \\
\qquad n=79\end{array}$ & $\begin{array}{l}\text { Poor metabolic control } \\
\qquad n=82\end{array}$ & $p$-value 1 & $p$-value 2 \\
\hline \multicolumn{7}{|l|}{ Age [years] } \\
\hline $35-44$ & $15^{\mathrm{A}, \mathrm{a}}(19.2)$ & $11^{\mathrm{B}}(6.8)$ & $7^{\mathrm{ab}}(8.9)$ & $4^{b}(4.9)$ & \multirow{3}{*}{0.000} & \multirow{3}{*}{0.001} \\
\hline $45-54$ & $40^{A, a}(51.3)$ & $61^{B}(37.9)$ & $29^{a}(36.7)$ & $32^{\mathrm{a}}(39)$ & & \\
\hline $55-65$ & $23^{A, a}(29.5)$ & $89^{B}(55.3)$ & $43^{b}(54.4)$ & $46^{\mathrm{b}}(56.1)$ & & \\
\hline \multicolumn{7}{|l|}{ Gender } \\
\hline female & $39^{\mathrm{A}, \mathrm{ab}}(50)$ & $90^{A}(55.9)$ & $53^{b}(67.1)$ & $37^{\mathrm{a}}(45.1)$ & \multirow{2}{*}{0.391} & \multirow{2}{*}{0.014} \\
\hline male & $39^{\mathrm{A}, \mathrm{ab}}(50)$ & $71^{A}(44.1)$ & $26^{b}(32.9)$ & $45^{\mathrm{a}}(54.9)$ & & \\
\hline \multicolumn{7}{|l|}{$\mathrm{BMI}\left[\mathrm{kg} / \mathrm{m}^{2}\right]$} \\
\hline normal (BMI < 25) & $40^{A, a}(51.3)$ & $9^{B}(5.6)$ & $6^{\mathrm{b}}(7.6)$ & $3^{b}(3.7)$ & \multirow{3}{*}{0.000} & \multirow{3}{*}{0.000} \\
\hline overweight $(25 \leq \mathrm{BMI}<30)$ & $36^{\mathrm{A}, \mathrm{a}}(46.2)$ & $127^{\mathrm{B}}(78.9)$ & $60^{b}(75.9)$ & $67^{b}(81.7)$ & & \\
\hline obese (BMI $\geq 30$ ) & $2^{\mathrm{A}, \mathrm{a}}(2.6)$ & $25^{B}(15.5)$ & $13^{b}(16.5)$ & $12^{b}(14.6)$ & & \\
\hline \multicolumn{7}{|l|}{ Education level } \\
\hline primary & $20^{A, a}(25.6)$ & $88^{B}(54.7)$ & $45^{b}(57)$ & $43^{b}(52.4)$ & \multirow{4}{*}{0.000} & \multirow{4}{*}{0.001} \\
\hline high school & $42^{A, a}(53.8)$ & $v 58^{B}(36.0)$ & $24^{b}(30.4)$ & $34^{\mathrm{ab}}(41.5)$ & & \\
\hline university & $14^{\mathrm{A}, \mathrm{a}}(17.9)$ & $12^{B}(7.5)$ & $7^{\mathrm{a}}(8.9)$ & $5^{\mathrm{a}}(6.1)$ & & \\
\hline postgraduate & $2^{A, a}(2.6)$ & $3^{A}(1.9)$ & $3^{\mathrm{a}}(3.8)$ & $0^{a}(0)$ & & \\
\hline \multicolumn{7}{|l|}{ Dental visit frequency } \\
\hline$\leq$ once a year & $4^{\mathrm{A}, \mathrm{a}}(5.1)$ & $143^{B}(88.8)$ & $67^{b}(84.8)$ & $76^{b}(92.7)$ & \multirow{3}{*}{0.000} & \multirow{3}{*}{0.000} \\
\hline once a year & $35^{\mathrm{A}, \mathrm{a}}(44.9)$ & $16^{\mathrm{B}}(9.9)$ & $11^{b}(13.9)$ & $5^{b}(6.1)$ & & \\
\hline$\geq$ twice a year & $39^{A, a}(50.0)$ & $2^{B}(1.2)$ & $1^{b}(1.3)$ & $1^{b}(1.2)$ & & \\
\hline \multicolumn{7}{|l|}{ Tooth brushing frequency } \\
\hline$\leq$ once a day & $9^{A, a}(11.5)$ & $116^{\mathrm{B}}(72.0)$ & $61^{b}(77.2)$ & $55^{b}(67.1)$ & \multirow{3}{*}{0.000} & \multirow{3}{*}{0.000} \\
\hline once a day & $32^{A, a}(41.0)$ & $38^{b}(23.6)$ & $15^{b}(19.0)$ & $23^{\mathrm{ab}}(28.0)$ & & \\
\hline$\geq$ twice a day & $37^{A, a}(47.4)$ & $7^{b}(4.3)$ & $3^{b}(3.8)$ & $4^{b}(4.9)$ & & \\
\hline \multicolumn{7}{|l|}{ Smoking } \\
\hline none & $63^{A, a}(80.8)$ & $106^{\mathrm{B}}(44.35)$ & $55^{\mathrm{ab}}(69.6)$ & $51^{b}(62.2)$ & \multirow{5}{*}{0.016} & \multirow{5}{*}{0.054} \\
\hline former & $12^{A, a}(15.4)$ & $22^{B}(9.2)$ & $10^{\mathrm{a}}(12.7)$ & $12^{a}(14.6)$ & & \\
\hline$<10$ cigarettes/day & $3^{\mathrm{A}, \mathrm{a}}(3.8)$ & $18^{B}(7.53)$ & $9^{\mathrm{a}}(11.4)$ & $9^{\mathrm{a}}(11.0)$ & & \\
\hline 10-20 cigarettes/day & $\mathrm{O}^{\mathrm{A}, \mathrm{a}}(0)$ & $13^{B}(5.4)$ & $4^{\mathrm{a} b}(5.1)$ & $g^{b}(11.0)$ & & \\
\hline >20 cigarettes/day & $0^{A, a}(0)$ & $2^{B}(0.8)$ & $1^{\mathrm{a}}(1.3)$ & $1^{\mathrm{a}}(1.2)$ & & \\
\hline \multicolumn{7}{|l|}{ Diabetes diagnosis age [years] } \\
\hline $0-4$ & & $69(42.9)$ & $36^{a}(45.6)$ & $33^{a}(40.2)$ & \multirow{3}{*}{-} & \multirow{3}{*}{-} \\
\hline $5-9$ & - & $60(37.3)$ & $30^{\mathrm{a}}(38)$ & $30^{\mathrm{a}}(36.6)$ & & \\
\hline$\geq 10$ & & $32(19.9)$ & $13^{\mathrm{a}}(16.5)$ & $19^{\mathrm{a}}(23.2)$ & & \\
\hline \multicolumn{7}{|l|}{ Drug use } \\
\hline oral antidiabetic & & $141(87.6)$ & $66^{\mathrm{a}}(83.5)$ & $75^{\mathrm{a}}(91.5)$ & & \\
\hline oral antidiabetic + insulin & & $20(12.4 \%)$ & $13^{\mathrm{a}}(16.5)$ & $7^{\mathrm{a}}(8.5 \%)$ & & \\
\hline antihypertensive & - & $26(16.1)$ & $14^{\mathrm{a}}(17.7)$ & $12^{a}(14.6)$ & - & - \\
\hline antilipemic & & $16(9.9)$ & $7^{\mathrm{a}}(8.9)$ & $9^{a}(11)$ & & \\
\hline cardiovascular & & $28(17.4)$ & $11^{\mathrm{a}}(13.9)$ & $17^{\mathrm{a}}(20.7)$ & & \\
\hline
\end{tabular}

Categorical variables are presented as $\mathrm{n}(\%)$. The $p$-values were computed with the $\mathrm{X}^{2}$ independence test. BMI - body mass index; $\mathrm{H}-$ healthy controls; T2DM type 2 diabetes mellitus patients. Capital letters and $p$-value 1 : significant difference between groups T2DM and H; small letters and $p$-value 2 : significant difference between group $\mathrm{H}$ and patients with good and poor metabolic control; different letters denote statistical significance at $p<0.05$ for each parameter after the least significant difference (LSD) multiple comparison test.

metabolic control $(p<0.05)$, good metabolic control did not lead to a significant decrease in adiponectin $(p<0.05)$. However, when covariates were adjusted, statistical differences in the IL-6 and adiponectin levels became insignificant and, furthermore, significantly higher vaspin levels in the T2DM patients, regardless 
Table 2. Comparisons of metabolic parameters according to metabolic control status

\begin{tabular}{|c|c|c|c|c|c|}
\hline Parameters & Metabolic control & $\mathrm{n}$ & Mean \pm SD & $p$-value 1 & $p$-value 2 \\
\hline \multirow{3}{*}{$\begin{array}{l}\text { FBS } \\
{[\mathrm{mg} / \mathrm{dL}]}\end{array}$} & healthy & 78 & $90.87 \pm 8.33^{\mathrm{a}, \mathrm{A}}$ & \multirow{3}{*}{0.000} & \multirow{3}{*}{0.000} \\
\hline & good & 79 & $127.73 \pm 16.56^{\mathrm{b}, \mathrm{B}}$ & & \\
\hline & poor & 82 & $174.45 \pm 38.08^{c, c}$ & & \\
\hline \multirow{3}{*}{$\begin{array}{l}\mathrm{HbA}_{1 \mathrm{c}} \\
{[\%]}\end{array}$} & healthy & 78 & $5.12 \pm 0.30^{\mathrm{a}, \mathrm{A}}$ & \multirow{3}{*}{0.000} & \multirow{3}{*}{0.000} \\
\hline & good & 79 & $6.44 \pm 0.40^{\mathrm{b}, \mathrm{B}}$ & & \\
\hline & poor & 82 & $7.63 \pm 0.41^{c, c}$ & & \\
\hline \multirow{3}{*}{$\begin{array}{l}\text { TC } \\
{[\mathrm{mg} / \mathrm{dL}]}\end{array}$} & healthy & 78 & $148.82 \pm 42.37^{\mathrm{a}, \mathrm{A}}$ & \multirow{3}{*}{0.000} & \multirow{3}{*}{0.236} \\
\hline & good & 79 & $173.86 \pm 45.32^{\mathrm{b}, \mathrm{A}}$ & & \\
\hline & poor & 82 & $183.86 \pm 54.04^{\mathrm{b}, \mathrm{A}}$ & & \\
\hline \multirow{3}{*}{$\begin{array}{l}\text { TG } \\
{[\mathrm{mg} / \mathrm{dL}]}\end{array}$} & healthy & 78 & $112.12 \pm 32.76^{\mathrm{a}, \mathrm{A}}$ & \multirow{3}{*}{0.000} & \multirow{3}{*}{0.260} \\
\hline & good & 79 & $158.83 \pm 62.72^{\mathrm{b}, \mathrm{A}}$ & & \\
\hline & poor & 82 & $169.10 \pm 88.33^{b, A}$ & & \\
\hline \multirow{3}{*}{$\begin{array}{l}\mathrm{HDL} \\
{[\mathrm{mg} / \mathrm{dL}]}\end{array}$} & healthy & 78 & $64.06 \pm 10.69^{\mathrm{a}, \mathrm{A}}$ & \multirow{3}{*}{0.000} & \multirow{3}{*}{0.000} \\
\hline & good & 79 & $47.37 \pm 9.68^{b, B}$ & & \\
\hline & poor & 82 & $45.61 \pm 9.16^{b, B}$ & & \\
\hline \multirow{3}{*}{$\begin{array}{l}\text { LDL } \\
{[\mathrm{mg} / \mathrm{dL}]}\end{array}$} & healthy & 78 & $107.43 \pm 20.47^{\mathrm{a}, \mathrm{A}}$ & \multirow{3}{*}{0.066} & \multirow{3}{*}{0.394} \\
\hline & good & 79 & $117.76 \pm 34.28^{b, A}$ & & \\
\hline & poor & 82 & $116.36 \pm 33.05^{\mathrm{ab}, \mathrm{A}}$ & & \\
\hline \multirow{3}{*}{$\mathrm{TC} / \mathrm{HDL}$} & healthy & 78 & $2.42 \pm 0.89^{a, A}$ & \multirow{3}{*}{0.000} & \multirow{3}{*}{0.001} \\
\hline & good & 79 & $3.75 \pm 0.98^{b, B}$ & & \\
\hline & poor & 82 & $4.12 \pm 1.26^{\mathrm{c}, \mathrm{B}}$ & & \\
\hline \multirow{3}{*}{$\begin{array}{l}\text { ALT } \\
{[U / L]}\end{array}$} & healthy & 78 & $22.40 \pm 11.60^{\mathrm{a}, \mathrm{A}}$ & \multirow{3}{*}{0.017} & \multirow{3}{*}{0.175} \\
\hline & good & 79 & $29.63 \pm 18.41^{b, A}$ & & \\
\hline & poor & 82 & $27.82 \pm 18.19^{b, A}$ & & \\
\hline \multirow{3}{*}{$\begin{array}{l}\text { Creatinine } \\
{[\mathrm{mg} / \mathrm{dL}]}\end{array}$} & healthy & 78 & $0.97 \pm 0.17^{\mathrm{a}, \mathrm{A}}$ & \multirow{3}{*}{0.913} & \\
\hline & good & 79 & $0.98 \pm 0.24^{\mathrm{a}, \mathrm{A}}$ & & 0.811 \\
\hline & poor & 82 & $0.99 \pm 0.24^{\mathrm{a}, \mathrm{A}}$ & & \\
\hline & healthy & 78 & $1.70 \pm 0.63^{\mathrm{a}, \mathrm{A}}$ & & \\
\hline NLR & good & 79 & $2.27 \pm 0.87^{b, B}$ & 0.000 & 0.001 \\
\hline & poor & 82 & $2.29 \pm 0.98^{b, B}$ & & \\
\hline & healthy & 78 & $3.33 \pm 0.98^{a, A}$ & & \\
\hline $\begin{array}{l}\text { CRP } \\
{[\mathrm{mg} / 1]}\end{array}$ & good & 79 & $4.39 \pm 2.2^{\mathrm{b}, \mathrm{A}}$ & 0.000 & 0.004 \\
\hline & poor & 82 & $4.97 \pm 2.02^{\mathrm{C}, \mathrm{B}}$ & & \\
\hline & healthy & 78 & $10.77 \pm 0.98^{\mathrm{a}, \mathrm{A}}$ & & \\
\hline $\begin{array}{l}\text { Systolic blood pressure } \\
{[\mathrm{mmHa}]}\end{array}$ & good & 79 & $12.35 \pm 0.75^{b, B}$ & 0.000 & 0.000 \\
\hline & poor & 82 & $12.32 \pm 0.84^{b, B}$ & & \\
\hline & healthy & 78 & $7.38 \pm 0.61^{\mathrm{a}, \mathrm{A}}$ & & \\
\hline $\begin{array}{l}\text { Diastolic blood pressure } \\
{[\mathrm{mmHa}]}\end{array}$ & good & 79 & $8.37 \pm 0.6^{b, B}$ & 0.000 & 0.000 \\
\hline & poor & 82 & $8.37 \pm 0.62^{b, B}$ & & \\
\hline
\end{tabular}

The $p$-values were computed by multivariate variance analysis (MANOVA). ALT - alanine aminotransferase; CRP - C-reactive protein; FBS - fasting blood sugar; $\mathrm{HbA}_{1 \mathrm{c}}$ - glycated hemoglobin A; HDL - high-density lipoprotein; LDL - low-density lipoprotein; NLR - neutrophil-to-lymphocyte ratio; SD - standard deviation; TC - total cholesterol. Small letters and p-value 1: significant difference when covariates (age, gender, BMI, education level, smoking, dental visit and tooth brushing frequency) unadjusted; capital letters and $p$-value 2: significant difference when covariates adjusted; different letters denote statistical significance at $p<0.05$ for each parameter after the LSD multiple comparison test.

of the metabolic control status, were noticed when compared with the $\mathrm{H}$ individuals $(p<0.05)$.

The significant correlations are presented in Table 4. In the $\mathrm{H}$ controls, there were significant positive correla- tions as follows: FBS-GI; TC-CAL; TC-BOP\%; ALT-GI; ALT-PPD; ALT-TC/HDL; number of missing teeth-salivary volume; and number of missing teeth-LDL. When adipokines were considered, TNF- $\alpha-I L-6$; TNF- $\alpha-C R P$; 
Table 3. Intraoral parameters and adipokines according to metabolic control status

\begin{tabular}{|c|c|c|c|c|c|}
\hline Parameters & Metabolic control & $\mathrm{n}$ & Mean \pm SD & $p$-value 1 & $p$-value 2 \\
\hline \multirow{3}{*}{$\mathrm{PI}$} & healthy & 78 & $1.79 \pm 0.40^{\mathrm{a}, \mathrm{A}}$ & \multirow{3}{*}{0.000} & \multirow{3}{*}{0.207} \\
\hline & good & 79 & $2.07 \pm 0.42^{b, A}$ & & \\
\hline & poor & 82 & $2.15 \pm 0.43^{b, A}$ & & \\
\hline \multirow{3}{*}{ Gl } & healthy & 78 & $1.60 \pm 0.40^{\mathrm{a}, \mathrm{A}}$ & \multirow{3}{*}{0.000} & \multirow{3}{*}{0.006} \\
\hline & good & 79 & $1.89 \pm 0.20^{b, B}$ & & \\
\hline & poor & 82 & $1.92 \pm 0.20^{b, B}$ & & \\
\hline \multirow{3}{*}{$\mathrm{BOP} \%$} & healthy & 78 & $59.66 \pm 35.14^{\mathrm{a}, \mathrm{A}}$ & \multirow{3}{*}{0.000} & \multirow{3}{*}{0.000} \\
\hline & good & 79 & $93.30 \pm 14.94^{\mathrm{b}, \mathrm{B}}$ & & \\
\hline & poor & 82 & $96.39 \pm 9.58^{b, B}$ & & \\
\hline \multirow{3}{*}{ Number of missing teeth } & healthy & 78 & $2.13 \pm 2.00^{\mathrm{a}, \mathrm{A}}$ & \multirow{3}{*}{0.000} & \multirow{3}{*}{0.002} \\
\hline & good & 79 & $4.57 \pm 2.55^{\mathrm{b}, \mathrm{B}}$ & & \\
\hline & poor & 82 & $3.94 \pm 2.80^{b, B}$ & & \\
\hline \multirow{3}{*}{$\begin{array}{l}\text { PPD } \\
{[\mathrm{mm}]}\end{array}$} & healthy & 78 & $3.02 \pm 0.72^{\mathrm{a}, \mathrm{A}}$ & \multirow{3}{*}{0.000} & \multirow{3}{*}{0.002} \\
\hline & good & 79 & $3.59 \pm 0.87^{b, A B}$ & & \\
\hline & poor & 82 & $3.92 \pm 0.84^{C, B}$ & & \\
\hline \multirow{3}{*}{$\begin{array}{l}\mathrm{CAL} \\
{[\mathrm{mm}]}\end{array}$} & healthy & 78 & $3.33 \pm 0.82^{\mathrm{a}, \mathrm{A}}$ & \multirow{3}{*}{0.000} & \multirow{3}{*}{0.004} \\
\hline & good & 79 & $3.90 \pm 0.82^{\mathrm{b}, \mathrm{AB}}$ & & \\
\hline & poor & 82 & $4.22 \pm 0.87^{c, B}$ & & \\
\hline \multirow{3}{*}{$\begin{array}{l}\text { PESA } \\
{\left[\mathrm{mm}^{2}\right]}\end{array}$} & healthy & 78 & $1773.83 \pm 565.13^{\mathrm{a}, \mathrm{A}}$ & \multirow{3}{*}{0.000} & \multirow{3}{*}{0.001} \\
\hline & good & 79 & $2194.12 \pm 574.61^{b, B}$ & & \\
\hline & poor & 82 & $2311.15 \pm 524.9^{b, B}$ & & \\
\hline \multirow{3}{*}{$\begin{array}{l}\text { PISA } \\
{\left[\mathrm{mm}^{2}\right]}\end{array}$} & healthy & 78 & $1236.47 \pm 707.84^{\mathrm{a}, \mathrm{A}}$ & \multirow{3}{*}{0.001} & \multirow{3}{*}{0.003} \\
\hline & good & 79 & $2077.93 \pm 2430.34^{b, B}$ & & \\
\hline & poor & 82 & $1992.91 \pm 663.62^{b, B}$ & & \\
\hline \multirow{3}{*}{ TDI } & healthy & 78 & $2.40 \pm 1.05^{\mathrm{a}, \mathrm{A}}$ & & \\
\hline & good & 79 & $4.67 \pm 1.43^{\mathrm{b}, \mathrm{B}}$ & 0.000 & 0.000 \\
\hline & poor & 82 & $4.62 \pm 1.48^{\mathrm{b}, \mathrm{B}}$ & & \\
\hline & healthy & 78 & $0.54 \pm 0.07^{\mathrm{a}, \mathrm{A}}$ & & \\
\hline $\begin{array}{l}\text { Salivary volume } \\
{[\mathrm{mL} / \mathrm{min}]}\end{array}$ & good & 79 & $0.44 \pm 0.05^{\mathrm{b}, \mathrm{B}}$ & 0.000 & 0.000 \\
\hline & poor & 82 & $0.44 \pm 0.06^{b, B}$ & & \\
\hline & healthy & 78 & $10.60 \pm 8.22^{\mathrm{a}, \mathrm{A}}$ & & \\
\hline $\begin{array}{l}\text { TNF-a } \\
{[\mathrm{pq} / \mathrm{mL}]}\end{array}$ & good & 79 & $21.70 \pm 18.18^{\mathrm{b}, \mathrm{B}}$ & 0.000 & 0.000 \\
\hline & poor & 82 & $21.02 \pm 17.60^{b, B}$ & & \\
\hline & healthy & 78 & $2.64 \pm 1.72^{\mathrm{a}, \mathrm{A}}$ & & \\
\hline $\begin{array}{l}\text { IL-6 } \\
{[\mathrm{pg} / \mathrm{mL}]}\end{array}$ & good & 79 & $3.60 \pm 2.74^{\mathrm{b}, \mathrm{A}}$ & 0.040 & 0.840 \\
\hline & poor & 82 & $3.50 \pm 3.10^{\mathrm{b}, \mathrm{A}}$ & & \\
\hline & healthy & 78 & $17.10 \pm 12.72^{\mathrm{a}, \mathrm{A}}$ & & \\
\hline Adiponectin & good & 79 & $13.88 \pm 10.85^{a b, A}$ & 0.026 & 0.517 \\
\hline & poor & 82 & $12.63 \pm 8.07^{b, A}$ & & \\
\hline & healthy & 78 & $931.43 \pm 695.04^{\mathrm{a}, \mathrm{A}}$ & & \\
\hline $\begin{array}{l}\text { Vaspin } \\
{[\mathrm{pg} / \mathrm{mL}]}\end{array}$ & good & 79 & $1116.37 \pm 471.45^{\mathrm{b}, \mathrm{B}}$ & 0.055 & 0.030 \\
\hline & poor & 82 & $1112.96 \pm 448.99^{b, B}$ & & \\
\hline
\end{tabular}

The $p$-values were computed by multivariate variance analysis (MANOVA). BOP\% - percentage of bleeding on probing; CAL - clinical attachment level; GI - gingival index; II-6 - interleukin 6; PESA - periodontal epithelial surface area; PI - plaque index; PISA - periodontal inflamed surface area; PPD - periodontal pocket depth; TDI - total dental index; TNF-a - tumor necrosis factor a. Small letters and $p$-value 1: significant difference when covariates (age, gender, BMI, education level, smoking, dental visit and tooth brushing frequency) unadjusted; capital letters and $p$-value 2: significant difference when covariates adjusted; different letters denote statistical significance at $p<0.05$ for each parameter after the LSD multiple comparison test. 
Table 4. Significant correlations

\begin{tabular}{|c|c|c|}
\hline Correlation & $r$ & $p$-value \\
\hline \multicolumn{3}{|l|}{ Group H } \\
\hline GI-FBS & $0.290^{* *}$ & 0.010 \\
\hline GI-ALT & $0.237^{*}$ & 0.037 \\
\hline $\mathrm{BOP} \%-\mathrm{TC}$ & $0.315^{* *}$ & 0.005 \\
\hline BOP\%-TK/HDL & $0.312^{* *}$ & 0.005 \\
\hline $\mathrm{BOP} \%-\mathrm{ALT}$ & $0.276^{* *}$ & 0.015 \\
\hline PPD-ALT & $0.291^{* *}$ & 0.010 \\
\hline CAL-TC & $0.227^{*}$ & 0.046 \\
\hline $\mathrm{CAL}-\mathrm{TC} / \mathrm{HDL}$ & $0.249^{*}$ & 0.028 \\
\hline CAL-ALT & $0.312^{* *}$ & 0.005 \\
\hline Number of missing teeth-LDL & $0.246^{*}$ & 0.030 \\
\hline Number of missing teeth-salivary volume & $0.240^{*}$ & 0.035 \\
\hline TNF-a-CRP & $0.240^{*}$ & 0.034 \\
\hline TNF-a-IL-6 & $0.284^{*}$ & 0.012 \\
\hline IL-6-NLR & $0.288^{*}$ & 0.010 \\
\hline IL-6-vaspin & $0.303^{* *}$ & 0.007 \\
\hline Adiponectin-NLR & $0.284^{*}$ & 0.012 \\
\hline Adiponectin-IL-6 & $0.240^{*}$ & 0.034 \\
\hline BMI-TC & $0.300^{* *}$ & 0.008 \\
\hline$B M I-L D L$ & $0.334^{* *}$ & 0.003 \\
\hline BMI-TC/HDL & $0.301^{* *}$ & 0.007 \\
\hline \multicolumn{3}{|l|}{ Group T2DM } \\
\hline PI-FBS & $0.157^{*}$ & 0.047 \\
\hline $\mathrm{PI}-\mathrm{CRP}$ & $0.207^{* *}$ & 0.009 \\
\hline BOP\%-FBS & $0.156^{*}$ & 0.048 \\
\hline PPD-HbA $1 c$ & $0.178^{*}$ & 0.024 \\
\hline CAL-FBS & $0.186^{*}$ & 0.018 \\
\hline $\mathrm{CAL}-\mathrm{HbA} 1 \mathrm{c}$ & $0.185^{*}$ & 0.019 \\
\hline$C A L-C R P$ & $0.190^{*}$ & 0.016 \\
\hline PISA-NLR & $0.262^{* *}$ & 0.001 \\
\hline Number of missing teeth- $\mathrm{HbA}_{1 \mathrm{c}}$ & $-0.172^{*}$ & 0.029 \\
\hline Number of missing teeth-creatinine & $0.176^{*}$ & 0.025 \\
\hline Number of missing teeth-CRP & $-0.173^{*}$ & 0.028 \\
\hline TNF-a-BOP\% & $0.173^{*}$ & 0.028 \\
\hline TNF-a-PPD & $0.195^{*}$ & 0.013 \\
\hline TNF-a-PESA & $0.223^{* *}$ & 0.004 \\
\hline TNF-a-PISA & $0.227^{* *}$ & 0.004 \\
\hline TNF-a-creatinine & $0.165^{*}$ & 0.037 \\
\hline IL-6-tooth brushing frequency & $-0.169^{*}$ & 0.032 \\
\hline IL-6-TNF-a & $0.193^{*}$ & 0.014 \\
\hline IL-6-vaspin & $-0.180^{*}$ & 0.022 \\
\hline Adiponectin-PISA & $0.227^{* *}$ & 0.001 \\
\hline Vaspin-tooth brushing frequency & $0.178^{*}$ & 0.024 \\
\hline Vaspin-TG & $0.227^{* *}$ & 0.004 \\
\hline Vaspin-LDL & $-0.250 * *$ & 0.001 \\
\hline Salivary volume-TDI & $-0.164^{*}$ & 0.037 \\
\hline Salivary volume-tooth brushing frequency & $0.229^{* *}$ & 0.003 \\
\hline
\end{tabular}

${ }^{*} p<0.05$; ** $p<0.01$.
IL-6-adiponectin, IL-6-vaspin; IL-6-NLR; and adiponectin-NLR were positively correlated. In addition, positive correlations were found between BMI and the lipid parameters (TC, LDL, TC/HLD).

In the T2DM patients, the following positive correlations were determined: FBS-PI; FBS-BOP\%; FBSCAL; $\mathrm{HbA}_{1 \mathrm{c}}-\mathrm{PPD} ; \mathrm{HbA}_{1 \mathrm{c}}-\mathrm{CAL}$; CRP-PI; CRP-CAL; and NLR-PISA. While negative correlations were found between the number of missing teeth and $\mathrm{HbA}_{1 \mathrm{c}}$, and the number of missing teeth and CRP, there were positive correlations between the number of missing teeth and creatinine, and the salivary volume and the tooth brushing frequency. Salivary TNF- $\alpha$ was positively related with $\mathrm{BOP} \%$, PPD, PESA, PISA, and creatinine. While the correlations IL-6-TNF- $\alpha$, vaspin-TG and vaspin-tooth brushing frequency were significantly positive, both vaspin-IL-6 and vaspin-LDL had negative correlations. In addition, there was a positive relationship between the salivary adiponectin levels and PISA, and a negative correlation between the salivary volume and TDI.

\section{Discussion}

A bidirectional relationship between periodontitis and T2DM, which are both chronic inflammatory diseases, is reported in many studies..$^{14}$ It has been suggested that cytokines released during chronic inflammation lead to the development of insulin resistance. ${ }^{1,15}$ Adipokines and cytokines secreted from adipose tissue regulate energy consumption, insulin resistance, inflammation, and wound healing. ${ }^{1,4}$ Adipokines have pro- (IL-6, TNF- $\alpha$, etc.) or anti-inflammatory (adiponectin, etc.) effects. ${ }^{1,4}$ If the factors released from the salivary glands participate in the inflammatory responses associated with T2DM, ${ }^{15}$ it offers an avenue for the treatment of this disease. The present research assessed whether the metabolic control status affects the levels of salivary adipokines in T2DM.

Clinical studies reported that patients with diabetes had more frequent and more severe periodontal disease than those without diabetes. ${ }^{1,5}$ Severe periodontitis makes metabolic control in T2DM more difficult. ${ }^{1}$ In the current investigation, all periodontal parameters were higher in the T2DM patients as compared with $\mathrm{H}$ controls. However, significant differences in CAL and PPD were only found between the T2DM patients with poor metabolic control and the $\mathrm{H}$ individuals when covariates were adjusted. The correlations $\mathrm{HbA}_{1 \mathrm{c}}-\mathrm{CAL}, \mathrm{HbA}_{1 \mathrm{c}}-\mathrm{PPD}$, FBS-CAL, and FBS-BOP\% in the patients with diabetes provided evidence that poor metabolic control had an adverse influence on periodontal health.

Periodontal inflamed surface area was higher in the T2DM patients than in the $\mathrm{H}$ controls. ${ }^{3}$ Nesse et al. suggested the existence of a dose-response relationship exists between the $\mathrm{HbA}_{1 \mathrm{c}}$ levels and PISA. ${ }^{2}$ Our findings did not 
reveal a significant correlation between $\mathrm{HbA}_{1 \mathrm{c}}$ and PISA in diabetics. ${ }^{3}$ However, a positive correlation between PISA and NLR supported the claim that periodontal inflammation could contribute to systemic inflammation in diabetics.

Oral complications, hyposalivation, infections, caries, and periodontal abscess, for example, increase due to poor metabolic control in T2DM patients. In diabetes, salivary hypofunction is revealed to be associated with drugs and poor metabolic control. In diabetes with poor metabolic control, the salivary volume decreases as compared with diabetes with good metabolic control and healthy individuals. ${ }^{8}$ In the present study, despite the fact that the salivary volume was lowered in the T2DM group, the metabolic control was ineffective. Hyposalivation in diabetes causes not only an increase in caries and periodontal disease, but also oral inflammation. Oikarinen et al. recorded higher TDI scores in patients with coronary artery disease and, moreover, diabetes was more common in those with coronary artery disease. ${ }^{16}$ Nylund et al. noticed that TDI scores increased in diabetes with nephropathy as compared with the patients with other chronic renal diseases. ${ }^{17}$ No participants in the current analysis had a high creatinine level and nephropathy. An increased oral inflammatory burden and higher TDI scores in the T2DM patients as compared with the $\mathrm{H}$ controls were consistent with a negative relationship between the salivary volume and TDI. There was no difference in the TDI scores between the T2DM patients with good and poor metabolic control.

The increases of BMI may disrupt the metabolic control. The BMI values in the subjects with diabetes were higher, but without any relation to the metabolic control, than those in the $\mathrm{H}$ individuals. In this study, only 1 patient had a BMI $>32$ and no individual was morbidly obese. The lipid parameters alter due to diabetes (increases in TC, TG and LDL, and a decrease in HDL) and despite good metabolic control, dyslipidemia often continues in T2DM patients. ${ }^{18}$ Laws and Reaven suggested that low HDL levels lead to hyperinsulinemia and insulin resistance. ${ }^{19}$ The increased TC/HDL ratio in relation to the impairment of the metabolic control found in the current work emphasized the importance of dyslipidemia in T2DM.

C-reactive protein has been associated with both periodontitis and T2DM. ${ }^{3,5}$ It has been suggested that CRP may contribute to insulin resistance by damaging intracellular signalling. ${ }^{5}$ In this study, the increased CRP levels in the T2DM patients were related to diabetes and impaired metabolic control. Also, CRP associated with CAL in T2DM revealed that periodontal disease impacted on systemic inflammation.

Adiponectin, an adipokine that has anti-inflammatory and anti-atherogenic effects, modulates glucose and lipid metabolism. ${ }^{6,20}$ The amelioration of the metabolic control related to diabetes has been reported to increase the serum adiponectin level. ${ }^{21}$ In this study, the T2DM patients had decreased salivary adiponectin levels as compared with the $\mathrm{H}$ controls, although there was no difference in the salivary adiponectin levels between good and poor metabolic control in diabetes. The salivary adiponectin level has not been associated with BMI in T2DM patients or controls. ${ }^{6,20}$ Although no significant differences in the serum adiponectin levels have been found between individuals with and without periodontitis, ${ }^{22}$ Zimmermann et al. reported that the serum adiponectin levels decrease in periodontitis. ${ }^{23}$ In the present study, when covariates were adjusted, the salivary adipokine levels were not statistically different among the groups. There was no relationship between salivary adiponectin and tooth brushing and the salivary adiponectin level was associated with PISA in T2DM, which is in accordance with the findings of Riis et al. ${ }^{20}$ Our results demonstrate that salivary adiponectin has beneficial functions in periodontal health, and the properties of salivary adiponectin may be different from serum adiponectin due to systemic or local inflammation. ${ }^{6}$

The adipokines TNF- $\alpha$ and IL- 6 , produced in adipose tissue, and are also cytokines that play an important role in tissue destruction in periodontitis. ${ }^{5,6}$ The overproduction of TNF- $\alpha$ induces inflammation and the death of pancreatic beta cells, which contributes to insulin resistance and T2DM pathogenesis. ${ }^{24}$ In the present study, higher TNF- $\alpha$ levels in T2DM patients and the relationships between the salivary TNF- $\alpha$ level and the periodontal parameters corroborated an increased severity of periodontal disease in patients with diabetes. Both TNF- $\alpha$ and IL- 6 have synergistic effects and increase the severity of periodontitis. ${ }^{5}$ The salivary IL-6 levels in T2DM patients are increased as compared with healthy controls, and this is related to the metabolic control. ${ }^{25}$ Although we found a positive correlation between the levels of TNF- $\alpha$ and IL- 6 in saliva, only the salivary TNF- $\alpha$ levels in patients with diabetes were prominently increased relative to the metabolic control. Increased salivary IL-6 in individuals with diabetes was not statistically different from the values obtained for the $\mathrm{H}$ controls.

Vaspin produced by adipose tissue, skeletal muscle, pancreas, and skin, has a regulatory role in glucose and lipid metabolism. ${ }^{7}$ The serum vaspin levels are reportedly higher in diabetes patients than in healthy controls, and also they are higher in diabetes with poor metabolic control as compared with diabetes with good metabolic control, ${ }^{7}$ although Yang et al. observed that vaspin decreased in T2DM. ${ }^{26}$ Balli et al. suggested that the gingival cervical fluid (GCF) vaspin levels increased in obese individuals with chronic periodontitis as compared with obese individuals without chronic periodontitis and non-obese individuals with chronic periodontitis. ${ }^{27}$ They found positive correlations between the GCF vaspin levels and the periodontal parameters, 
and also between the GCF vaspin levels and BMI. ${ }^{27}$ In our study, the low distribution of individuals with diabetes over 10 years in the T2DM group may have caused the vaspin levels to be independent of the metabolic control. Based on a review of the current literature, this is the first study to evaluate the salivary vaspin levels. In the T2DM patients, the increased salivary vaspin levels were not related to the metabolic control when compared with the $\mathrm{H}$ group. Although we did not detect any significant association between salivary vaspin and BMI, and salivary vaspin and the periodontal parameters in the $\mathrm{H}$ individuals, the protective role of oral vaspin was supported by the vaspin-tooth brushing frequency parallelism in the T2DM patients. In the T2DM group, the positive correlation between TG and salivary vaspin was compatible with previous findings, ${ }^{28}$ while a negative correlation appeared between LDL and salivary vaspin. Also, there were negative correlations between salivary IL-6 and vaspin in both groups (T2DM and H). Insulin and anti-diabetic drugs have brought the vaspin levels in patients with diabetes to normal. ${ }^{29}$ Statins increase the vaspin levels in serum. ${ }^{30}$ In this study, the T2DM patients had a decreased salivary volume. Microvascular changes and inflammatory tissue destruction caused by T2DM may result in structural degeneration in periodontium and other organs. ${ }^{1}$ Moreover, some T2DM patients take some medicines together; however, the precise effects of drugs on the salivary adipokine levels are not yet known.

\section{Conclusions}

Metabolic control in T2DM should be considered a conflicting factor in the relationship between periodontal and systemic diseases. Higher periodontal parameters in the T2DM patients than in the $\mathrm{H}$ controls are the evidence that metabolic control has a significant influence on periodontal health. This study is the first to evaluate the vaspin levels in saliva in relation to the metabolic control in T2DM patients. The levels of salivary adipokines were changed by T2DM, while being independent from the metabolic control. The salivary TNF- $\alpha$ and vaspin increases in the T2DM patients were significant as compared with the $\mathrm{H}$ individuals when covariates were adjusted. However, there was only 1 patient with a $\mathrm{BMI}>32$, no individuals had $\mathrm{HbA}_{1 \mathrm{c}}>8.5$, and none of the individuals had a mean $\mathrm{CAL}<6 \mathrm{~mm}$. Therefore, more severe impairment of the metabolic control in diabetes may influence the salivary adipokine levels. Also, the association between increased periodontal breakdown and more impaired metabolic control may contribute to altering the adipokine levels in saliva and cause a prominent difference in the adipokine levels between T2DM patients with poor and good metabolic control.

\section{ORCID iDs}

Elif Teke (1) https://orcid.org/0000-0001-5565-3541

Fatma Yeşim Kırzıoğlu (1) https://orcid.org/0000-0002-5240-4504

Hakan Korkmaz (10) https://orcid.org/0000-0001-5066-6335

Mustafa Calapoğlu (1) https://orcid.org/0000-0002-6539-1335

Hikmet Orhan (10) https://orcid.org/0000-0002-8389-1069

\section{References}

1. Mealey BL, Oates TW; American Academy of Periodontology. Diabetes mellitus and periodontal diseases. J Periodontol. 2006;77(8):1289-1303.

2. Nesse W, Linde A, Abbas F, et al. Dose-response relationship between periodontal inflamed surface area and $\mathrm{HbA1c}$ in type 2 diabetics. J Clin Periodontol. 2009;36(4):295-300.

3. Susanto H, Nesse W, Dijkstra PU, et al. Periodontal inflamed surface area and $\mathrm{C}$-reactive protein as predictors of $\mathrm{HbA}_{1 \mathrm{c}}$ : A study in Indonesia. Clin Oral Investig. 2012;16(4):1237-1242.

4. Sun WL, Chen LL, Zhang SZ, Ren YZ, Qin GM. Changes of adiponectin and inflammatory cytokines after periodontal intervention in type 2 diabetes patients with periodontitis. Arch Oral Biol. 2010;55(12):970-974.

5. Preshaw PM, Alba AL, Herrera D, et al. Periodontitis and diabetes: A two-way relationship. Diabetologia. 2012;55(1):21-31.

6. Li L, Wu LL. Adiponectin and interleukin- 6 in inflammation-associated disease. Vitam Horm. 2012;90:375-395.

7. Blüher M. Vaspin in obesity and diabetes: Pathophysiological and clinical significance. Endocrine. 2012;41(2):176-182.

8. von Bültzingslöwen I, Sollecito TP, Fox PC, et al. Salivary dysfunction associated with systemic diseases: Systematic review and clinical management recommendations. Oral Surg Oral Med Oral Pathol Oral Radiol Endod. 2007;103(Suppl S57):e1-15.

9. American Diabetes Association. 2. Classification and Diagnosis of Diabetes. Diab Care. 2017;40(Suppl 1):S11-24.

10. Silness J, Löe H. Periodontal disease in pregnancy. II. Correlation between oral hygiene and periodontal condition. Acta Odontol Scand. 1964;22(1):121-135.

11. Löe $\mathrm{H}$, Silness J. Periodontal disease in pregnancy. I. Prevalence and severity. Acta Odontol Scand. 1963;21(6):533-551.

12. Mattila KJ, Nieminen MS, Valtonen VV, et al. Association between dental health and acute myocardial infarction. BMJ. 1989;298(6676):779-781.

13. Navazesh M. Methods for collecting saliva. Ann N Y Acad Sci. 1993;694:72-77.

14. Casanova L, Hughes FJ, Preshaw PM. Diabetes and periodontal disease: A two-way relationship. Br Dent J. 2014;217(8):433-437.

15. Yin J, Gao H, Yang J, Xu L, Li M. Measurement of salivary resistin level in patients with type 2 diabetes. Int J Endocrinol. 2012;2012:359724.

16. Oikarinen K, Zubaid M, Thalib L, Soikkonen K, Rashed W, Lie T. Infectious dental diseases in patients with coronary artery disease: An orthopantomographic case-control study. J Can Dent Assoc. 2009;75(1):35.

17. Nylund KM, Meurman JH, Heikkinen AM, et al. Periodontal inflammatory burden and salivary matrix metalloproteinase- 8 concentration among patients with chronic kidney disease at the predialysis stage. J Periodontol. 2015;86(11):1212-1220.

18. Zhou X, Zhang W, Liu X, Zhang W, Li Y. Interrelationship between diabetes and periodontitis: Role of hyperlipidemia. Arch Oral Biol. 2015;60(4):667-674.

19. Laws A, Reaven G. Evidence for an independent relationship between insulin resistance and fasting plasma HDL-cholesterol, triglyceride and insulin concentrations. J Intern Med. 1992;231(1):25-30.

20. Riis JL, Bryce $\mathrm{Cl}, \mathrm{Ha} \mathrm{T}$, et al. Adiponectin: Serum-saliva associations and relations with oral and systemic markers of inflammation. Peptides. 2017;91:58-64.

21. Sun WL, Chen LL, Zhang SZ, Wu YM, Ren YZ, Qin GM. Inflammatory cytokines, adiponectin, insulin resistance and metabolic control after periodontal intervention in patients with type 2 diabetes and chronic periodontitis. Intern Med. 2011;50(15):1569-1574.

22. Furugen $\mathrm{R}$, Hayashida $\mathrm{H}$, Yamaguchi $\mathrm{N}$, et al. The relationship between periodontal condition and serum levels of resistin and adiponectin in elderly Japanese. J Periodontal Res. 2008;43(5):556-562. 
23. Zimmermann GS, Bastos MF, Dias Gonçalves TE, Chambrone L, Duarte PM. Local and circulating levels of adipocytokines in obese and normal weight individuals with chronic periodontitis. J Periodontol. 2013;84(5):624-633.

24. Mahmoud F, Al-Ozairi E. Inflammatory cytokines and the risk of cardiovascular complications in type 2 diabetes. Dis Markers. 2013;35(4):235-241.

25. Costa PP, Trevisan GL, Macedo GO, et al. Salivary interleukin-6, matrix metalloproteinase-8, and osteoprotegerin in patients with periodontitis and diabetes. J Periodontol. 2010;81(3):384-391.

26. Yang $W$, Li Y, Tian T, Wang L, Lee $P$, Hua $Q$. Serum vaspin concentration in elderly patients with type 2 diabetes mellitus and macrovascular complications. BMC Endocr Dis. 2017;17:67.

27. Balli U, Bozkurt Dogan S, Ongoz Dede F, Sertoglu E, Keles GC. The levels of visceral adipose tissue-derived serpin, omentin-1 and tumor necrosis factor-a in the gingival crevicular fluid of obese patients following periodontal therapy. J Oral Sci. 2016;58(4):465-473.

28. Suleymanoglu S, Tascilar E, Pirgon O, Tapan S, Meral C, Abaci A. Vaspin and its correlation with insulin sensitivity indices in obese children. Diabetes Res Clin Pract. 2009;84(3):325-328.

29. Klöting $N$, Kovacs $P$, Kern $M$, et al. Central vaspin administration acutely reduces food intake and has sustained blood glucose-lowering effects. Diabetologia. 2011;54(7):1819-1823.

30. Al-Azzam SI, Alzoubi KH, Abeeleh JA, Mhaidat NM, Abu-Abeeleh M. Effect of statin therapy on vaspin levels in type 2 diabetic patients. Clin Pharmacol. 2013;5:33-38. 